Sports Training

\title{
Official matches and training sessions: physiological demands of elite junior badminton players
}

\author{
Karen Christie Gomes Sales ${ }^{1}$ (D), Marcos Antônio Pereira Santos ${ }^{2}$ (D), Fabio Yuzo Nakamura ${ }^{3}$ (D), \\ Valmir Oliveira Silvino ${ }^{2}$ (D), Alyson Felipe da Costa Sena ${ }^{1}$ (D), Sérgio Luís Galan Ribeiro ${ }^{2}$ (D), \\ Jefferson Fernando Coelho Rodrigues Júnior ${ }^{1}$ (D), Christian Emmanuel Torres Cabido ${ }^{1}$ (D), \\ Thiago Teixeira Mendes ${ }^{1}$ \\ ${ }^{1}$ Universidade Federal do Maranhão, Departamento de Educação Física, São Luís, MA, Brasil; \\ ${ }^{2}$ Universidade Federal do Piauí, Núcleo de Estudo em Fisiologia do Exercício Aplicado ao \\ Desempenho e Saúde, Departamento de Biofisica e Fisiologia, Teresina, PI, Brasil; \\ ${ }^{3}$ Universidade Federal da Paraíba, Departamento de Educação Física, João Pessoa, PB, Brasil.
}

Associate Editor: Ricardo Augusto Barbieri, Assistant Professor at the Estácio UniSEB, Ribeirão Preto, SP, Brasil.

\begin{abstract}
Aim: To evaluate the intensity of training and official badminton matches (international championship) in junior elite players. Methods: Twelve elite players from the Brazilian junior national team (6 male and 6 female) were monitored during 19 training sessions and 50 official men's and women's single and double matches in the XXVII PanAmerican Junior Games. Subjects underwent an incremental running step test to determine maximal oxygen uptake $\left(\mathrm{VO}_{2} \mathrm{max}\right)$, and an individual relationship between heart rate $(\mathrm{HR})$ and $\mathrm{VO}_{2}$ max was established to estimate exercise intensity and oxygen uptake at official matches (OMs) and training sessions. HR was monitored during multi-shuttlecock (MS), technical-tactical (TT), and physical (PS) training methods, as well as during simulated matches (SMs) and OMs. Variables such as \%HRmax, $\mathrm{HR}$ zones, $\% \mathrm{VO}_{2} \mathrm{max}$, and energy expenditure were also calculated. Results: OM was the most intense activity monitored for male and female athletes, followed by SM according to \%HRmax $(86.8 \pm$ $4.1 \%$ and $84.4 \pm 5.0 \%$ for female and male, respectively, in OM vs. $74.6 \pm 2.3 \%$ and $75.0 \pm 5.0 \%$ for female and male, respectively, in SM). OM mean energy expenditure was $10.7 \pm 0.5 \mathrm{kcal} . \mathrm{min}-1$ for females and $14.9 \pm 4.6 \mathrm{kcal}^{\mathrm{min}} \mathrm{m}^{-1}$ for males. Conclusion: MS training has less physiological demand and OM presented higher intensity. SM, on the other hand, had the closest physiological demand to an OM.
\end{abstract}

Keywords: Racquet sports, heart rate, energy consumption, physical education and training, oxygen consumption.

\section{Introduction}

Badminton has greatly gained in popularity since its inclusion in the 1992 Olympic Games in Barcelona, and, according to the World Badminton Federation, around 200 million people play badminton worldwide ${ }^{1}$. The most common forms of the game are men's singles, women's singles, men's doubles, women's doubles, and mixed doubles. Athletes usually move, run, jump, and attack with the racket at high speed and intensity ${ }^{2,3}$. As it is characterized by actions of short duration, high intensity, and a short resting time at a high level of play, players must display specific technical and physical conditions ${ }^{4}$. A longitudinal study assessing official matches (OMs) from the Olympic Badminton Men's singles finals (1992-2012) verified a change in the temporal structure of the match. There was an increase in rally time, resting time, number of shots per rally, and shots frequency (34.0\%), as well as a decrease in the effective playing time $(-34.5 \%)^{5}$. Therefore, the game changed over the years, increasing the metabolic demands and requiring a greater resting time.

Badminton players make use of several training methods in their routine, such as multi-shuttlecock (MS), technical-tactical (TT), physical (PS), and simulated matches (SM). Thus, many training programmes were created in order to improve the fitness parameters of badminton players with the inclusion of strength, endurance, and flexibility exercises ${ }^{6,7}$.

The physiological demands in badminton have been largely investigated over the years. A literature review assessed the demands in adult badminton athletes during $\mathrm{OM}, \mathrm{SM}$, and MP, as well as dehydration and internal and external loads ${ }^{3}$. The authors evaluated anthropometric measures, somatotype, lactate blood levels, maximal oxygen consumption $\left(\mathrm{VO}_{2} \mathrm{max}\right)$, and maximal heart rate (HRmax). Similarly, other studies evaluated the oxygen consumption $\left(\mathrm{VO}_{2}\right)$, blood lactate level, and \%HRmax 
reached during $\mathrm{OM}^{8}$, as well as agility, coordination, speed, and $\mathrm{VO}_{2} \max ^{9}$ in junior elite badminton players. However, no articles comparing the demands of MS, TT, $\mathrm{SM}$, and $\mathrm{OM}$ in junior elite players were found.

Considering that the analysis of training and $\mathrm{OM}$ intensities is key to unravel the demands of a sport, further studies are recommended to better elucidate these features in badminton junior elite players. Previous studies investigated the intensity in other sports by assessing the percentage of HRmax (\%HRmax), percentage of $\mathrm{VO}_{2} \max ^{10-14}$, and energy expenditure (EE) ${ }^{15-17}$. In spite of the popularity of badminton, few researchers have investigated the match analysis characteristics of the sport ${ }^{5}$. Studies analyzing the intensity of training and $\mathrm{OM}$ in badminton junior elite athletes are scarce. This would provide important information regarding load control, programming, and performance assessment. Therefore, this study aimed to determine the intensity of training and OM play of badminton in junior elite athletes (international championship) through the analysis of physiological variables including $\% \mathrm{HRmax}, \mathrm{VO}_{2} \max$, and $\mathrm{EE}$.

\section{Material and methods}

\section{Subjects}

Six male $(14.4 \pm 2.1$ years, $167.1 \pm 11.9 \mathrm{~cm}, 56.3 \pm$ $12.3 \mathrm{~kg}, 9.3 \pm 2.1 \%$ body fat, $52.8 \pm 2.8 \mathrm{~mL} \cdot \mathrm{kg} \cdot \mathrm{min}^{-1}$, HRmax $202 \pm 9.0 \mathrm{bpm})$ and six female $(15.7 \pm 1.7$ years, $159.7 \pm 5.6 \mathrm{~cm}, 53.7 \pm 5.8 \mathrm{~kg}, 15.2 \pm 4.3 \%$ body fat, 45.3 $\pm 2.2 \mathrm{~mL} . \mathrm{kg} \cdot \mathrm{min}^{-1}$, HRmax $198 \pm 6.2 \mathrm{bpm}$ ) junior elite badminton athletes volunteered to participate in the study. All athletes played for the Brazilian national badminton team. Athletes were non-probabilistically and intentionally recruited when the following inclusion criteria were met: participate in regular systematic training six days a week (plus competitive matches) and compete in events sanctioned by the Brazilian Badminton Confederation.

This study was approved by the ethics committee of the Universidade Federal do Maranhão (UFMA), São Luís, MA, Brazil, under protocol 90938518.0.0000.5087. All participants and their legal representatives were informed about the characteristics of the study and they signed an informed consent form beforehand, in accordance with resolution 466/2012 of the National Health Council.

\section{Design}

This is a descriptive cross-sectional study. Data were collected during the specific training period, in which the athletes trained six times a week either in the morning or in the afternoon. The training sessions were subdivided into MS, TT, SM, and PS training methods. The MS consisted of training sessions in which the athlete would hit the shuttlecock with the racket after being randomly thrown by the coach. The TT included motor actions present in in-game situations. The athletes played SM against themselves. PS consisted of strength, power, and speed training. Additionally, the volunteers played OMs in the XXVII Pan-American Junior Games in Salvador, BA, Brazil. The training sessions used to assess the physiological demands of the athletes were carried out before the competition in which the OM occurred.

\section{Methodology}

Anthropometric measurements (body height and mass) and a progressive treadmill test were administered to each volunteer. Afterward, they were evaluated during 19 training sessions with an average duration of $190 \pm$ $10 \mathrm{~min}$ for the female players and $201 \pm 9 \mathrm{~min}$ for the male players. The breaks during the training used for feedback instructions, eating, and drinking were also considered. Moreover, the volunteers were monitored in 50 OMs, which took place during the XXVII Pan-American Junior Games in Salvador, BA, Brazil. Of these, there were 12 men's singles, 17 women's singles, 3 men's doubles, and 18 women's doubles matches.

Body mass was assessed using a digital scale (Welmy, model W300, Brazil) with an accuracy of 0.02 grams and a capacity of $150 \mathrm{~kg}$. Height was measured using a portable stadiometer attached to the scale, with an accuracy of $0.1 \mathrm{~cm}$. Body fat was estimated through the four-site skinfolds method proposed by Lohman ${ }^{18}$ using a scientific skinfold caliper (Sanny, USA, pressure of $10 \mathrm{~mm}^{2}$, precision of $0.1 \mathrm{~mm}$ ).

The athletes completed a progressive multistage incremental test on a motorized treadmill (TRG Fitness, Brazil) to measure $\mathrm{VO}_{2}$ and $\mathrm{HR}$ over all stages to establish a relationship between $\mathrm{VO}_{2}$ and $\mathrm{HR}$ and to determine $\mathrm{VO}_{2} \mathrm{max}$. The speed started at $6 \mathrm{~km} / \mathrm{h}$ at an initial $1 \%$ inclination and was gradually increased every $5 \mathrm{~min}$ by $2 \mathrm{~km} / \mathrm{h}$ until they reached $12 \mathrm{~km} / \mathrm{h}$. The interval between the stages was $2 \mathrm{~min}$ for the least intense stage (6 and $8 \mathrm{~km} / \mathrm{h}$ ) and $5 \mathrm{~min}$ for the most intense stage (10 and $12 \mathrm{~km} / \mathrm{h}$ ). In the second part of the protocol, the speed of $12 \mathrm{~km} / \mathrm{h}$ has remained and $2 \%$ inclination was gradually added every 2 min until exhaustion ${ }^{17}$. Before each test, the participants rested in a seated position on a chair $(5 \mathrm{~min})$ while the equipment used to measure $\mathrm{O}_{2}$ uptake and the HR was set up. Ventilation variables were measured, breath by breath, using a gas analyzer (K5, Cosmed, Italy) calibrated before each test according to the manufacturer's recommendations using a standard concentration gas mixture and a 3-L calibration syringe.

$\mathrm{VO}_{2}$ data were obtained during the progressive testing session. $\mathrm{VO}_{2} \max$ and $\mathrm{HRmax}$ values found were used in this investigation. A linear regression equation was used for each athlete utilizing the average values in the last min of each stage as proposed elsewhere ${ }^{19}$. The correlation between $\mathrm{HR}$ and $\mathrm{VO}_{2}$ was $\mathrm{r}=0.97 \pm 0.05$ for the males 
(ranging from 0.87 to 1.0 ) and $r=0.90 \pm 0.15$ for the females (from 0.61 to 0.99 ). Thus, $\mathrm{VO}_{2}$ was estimated for each training method and OM. Next, the EE (kcal. $\left.\mathrm{min}^{-1}\right)$ of the training sessions and OM was calculated by converting the total $\mathrm{VO}_{2}$ into $\mathrm{kcal} / \mathrm{min}$, establishing the value of $4.8 \mathrm{kcal} / 1 \mathrm{O}_{2}{ }^{20}$.

HR was recorded using the Team Pod@ Heart Monitor (Firstbeat, Finland) connected to the Firstbeat SPORTS Individual® software (Finland). A sensor was attached to the chest of the athletes with an elastic strap, which interacted with the telemetry receptor connected to the software in real-time. Five HR zones were established to describe each player's individual internal load: Zone 1, at $<60 \%$ HRmax; Zone 2, at $60-70 \%$ HRmax; Zone 3, at 70-80\% HRmax; Zone 4, at 80-90\% HRmax; and Zone 5, at $90-100 \% \mathrm{HRmax}^{21}$.

The dehydration percentage was analyzed based on the difference between body mass at the beginning and end of each training session and that of the OM. As it was calculated using the difference between pre-and post-exercise mass, this method implies that 1 gram of lost body mass is equivalent to $1 \mathrm{~mL}$ of lost water $^{22}$. During all training sessions and the OMs, the participants could drink water ad libitum. The ambient temperature and relative humidity were recorded every 15 min using a humidity psychrometer with an infrared thermometer (Extech MO297).

\section{Statistical analysis}

The normality and homogeneity of the data variance were analyzed via Shapiro-Wilk and Levene tests, respec- tively. The data are presented as the mean \pm standard deviation of the mean. Repeated one-way ANOVA measures were used to verify differences between \%HRmax, $\% \mathrm{VO}_{2} \max , \mathrm{EE}$, and $\mathrm{HR}$ zones for the training sessions and OMs. Tukey's posthoc test was used to identify which comparisons were different from each other. Repeated two-way ANOVA measures were used for male and female subjects under the different situations, followed by Tukey's posthoc test. Pearson correlation was used to examine the association between $\mathrm{HR}$ and $\mathrm{VO}_{2}$. A criterion alpha level of $p \leq 0.05$ was used to determine statistical significance. For each comparison, eta-squared $\left(\eta^{2}\right)$ was calculated as a measure of effect size. Values of $0.01,0.06$, and above 0.15 were considered as a small, medium, and large, respectively ${ }^{23}$. The entire statistical analysis was performed using the SigmaPlot version 11.0 statistical software.

\section{Results}

\section{Intensity of training and official matches}

The percentage of the HRmax in the female athletes during $\mathrm{OM}$ was higher than those of the training methods analyzed $\left(\mathrm{p}<0.001, \eta^{2}=0.8\right)$. MS had the smallest $\%$ HRmax. On the other hand, SM had a higher \%HRmax than TT $\left(\mathrm{p}<0.001, \eta^{2}=0.7\right)$. For the male athletes, OM had higher \%HRmax than the training methods analyzed $\left(\mathrm{p}<0.009, \eta^{2}=0.8\right)$, PS was superior to MS $(\mathrm{p}=0.005$, $\eta^{2}=0.7$ ), and SM had higher values than MS and TT $\left(\mathrm{p}<0.048, \eta^{2}=0.8\right)$. No differences between males and
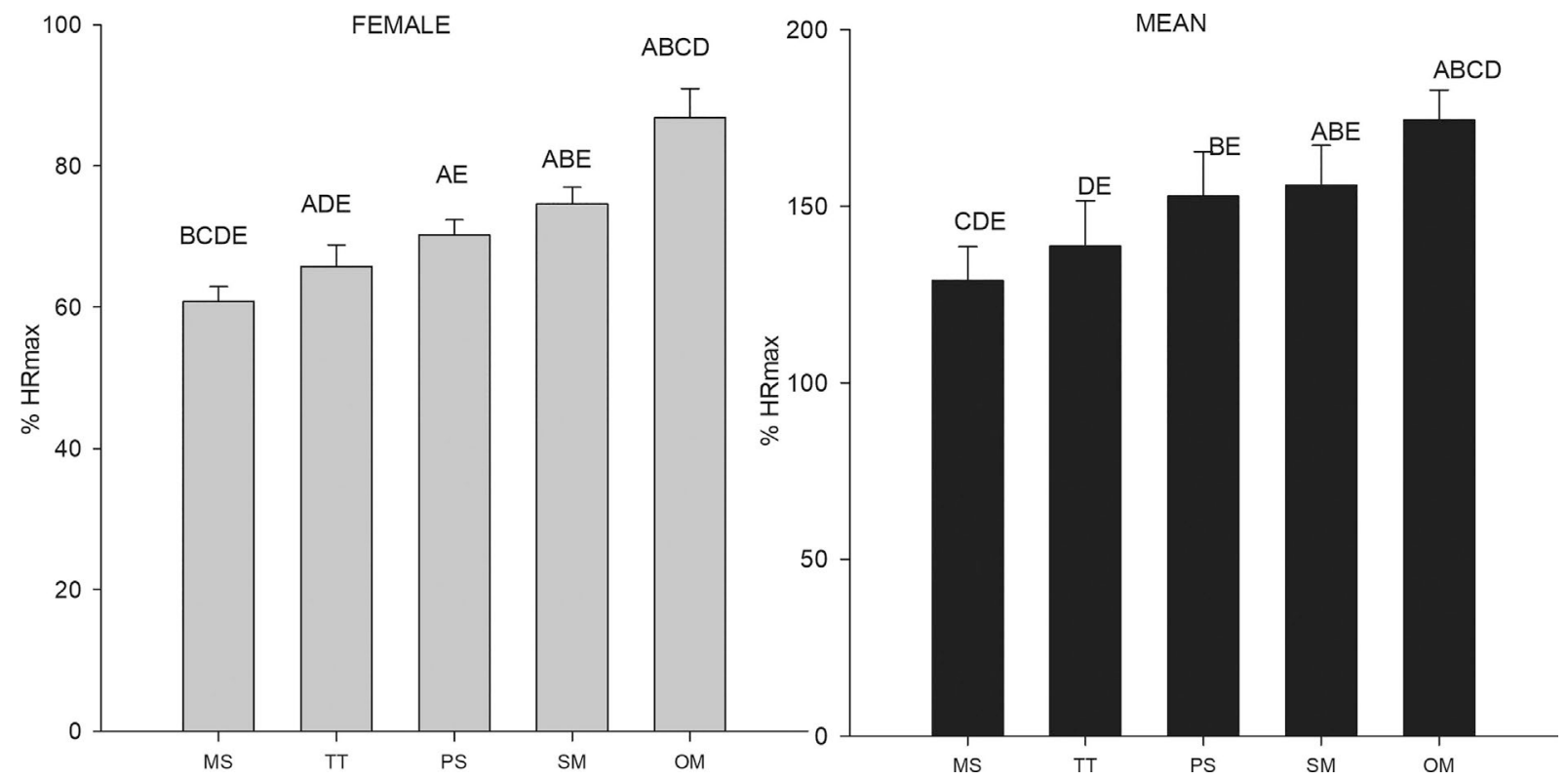

Figure 1 - \%HRmax reached in in in the multi-shuttlecock, technical-tactical, physical, simulated match, and official match for the female and male athletes. A, significant difference compared to multi-shuttlecock; B, significant difference compared to technical-tactical; C, significant difference compared to physical; D, significant difference compared to simulated match $(\mathrm{p}<0.05)$. 
females were observed for the \%HRmax $(p=0.9$, $\left.\eta^{2}=0.99\right)$ (Figure 1).

Regarding the aerobic demand of the female players, $\mathrm{OM}$ had higher $\% \mathrm{VO}_{2}$ max values compared to the training methods $\left(\mathrm{p}<0.001, \eta^{2}=0.76\right)$, PS and SM were higher than MS $\left(p<0.003, \eta^{2}=0.78\right)$. For the male players, the only significant difference found was the higher \% $\mathrm{VO}_{2}$ max in OM compared to MS and TT ( $\mathrm{p}<0.005$, $\left.\eta^{2}=0.34\right)$. There was no significant difference when comparing male and female athletes $\left(p=0.5, \eta^{2}=0.76\right)$ (Figure 2).

Durations of exercise at each HR zone are shown in Figure 3 Female athletes spent more time in zone 1 than in zones 3,4 , and $5\left(\mathrm{p}<0.047, \eta^{2}>0.54\right)$. Time spent in zone 2 was higher than in zones 4 and $5(\mathrm{p}<0.01$, $\left.\eta^{2}>0.54\right)$, and zone 3 was longer than zone $5(p=0.007$, $\left.\eta^{2}>0.54\right)$. In contrast, the male players remained for a longer time in zone 1 than in zones 3,4 , and $5(\mathrm{p}<0.002$, $\left.\eta^{2}>0.84\right)$, and zone 2 was longer than zone $5(p=0.001$, $\eta^{2}>0.52$ ). There was no significant difference between male and female athletes $\left(\mathrm{p}<0.881, \eta^{2}>0.82\right)$.

During the TT, the female athletes spent more time in zones 1 and 2 compared to zones 4 and $5(\mathrm{p}<0.021$, $\eta^{2}>0.72$ ). Zone 3 time was longer than that of zone 5 $\left(p=0.002, \eta^{2}>0.72\right)$. The male players spent more time in zones 1 and 2 compared to zones 4 and $5(\mathrm{p}<0.05$, $\left.\eta^{2}>0.72\right)$, and zone 3 was longer than zone $5(p=0.01$, $\left.\eta^{2}>0.73\right)$. No significant difference was observed between male and female athletes $\left(p<0.3, \eta^{2}>0.72\right)$ (Figure 3).

During the PS, female athletes spent more time in zones $1,2,3$, and 4 compared to zone $5(\mathrm{p}<0.003$, $\left.\eta^{2}=0.26\right)$, and zone 2 time was higher than that of zone 1 $\left(p=0.03, \eta^{2}=0.22\right)$. On the other hand, there was no difference in time between zones for the male players. No difference was observed between male and female athletes $\left(\mathrm{p}>0.05, \eta^{2}=0.22\right)$.

During the SM, female players spent more time in zones 3 and 4 compared to zones 1,2 , and 5 ( $p<0.006$, $\left.\eta^{2}=0.39\right)$. Similarly, zone 2 time was higher than that of zones 1 and $5\left(p<0.01, \eta^{2}>0.34\right)$. In addition, there was no difference in time between zones for the male players. No difference was observed between male and female athletes $\left(\mathrm{p}<0.05, \eta^{2}=0.22\right)$.

In OM, female athletes spent more time in zone 5 compared to zones 1,2 , and $3\left(\mathrm{p}<0.02, \eta^{2}=0.42\right)$. Zone 4 time was higher than that of zones 1 and $2(p<0.02$, $\left.\eta^{2}=0.53\right)$. The male players remained for longer in zones 4 and 5 compared to zone $1\left(p<0.05, \eta^{2}=0.52\right)$. No difference was observed between male and female athletes $\left(\mathrm{p}=0.4, \eta^{2}=0.22\right)$.

\section{Energy expenditure}

Table 1 shows the EE in different situations. Female players spent more energy during OMs than in the training methods $\left(p<0.001, \eta^{2}=0.1\right)$. PS and OM had more EE than MS $\left(p<0.002, \eta^{2}=0.77\right)$. Male players spent more energy during OM than in MS, TT, and PS $(\mathrm{p}<0.03$, $\left.\eta^{2}=0.77\right)$. EE during SM was higher than that of MS $\left(p=0.01, \eta^{2}=0.1\right)$. No significant difference was observed between male and female players $(\mathrm{p}=0.06$, $\left.\eta^{2}=0.1\right)$.

Air humidity, ambient temperature, and $<2 \%$ dehydration, which are well known to impair physical
FEMALE

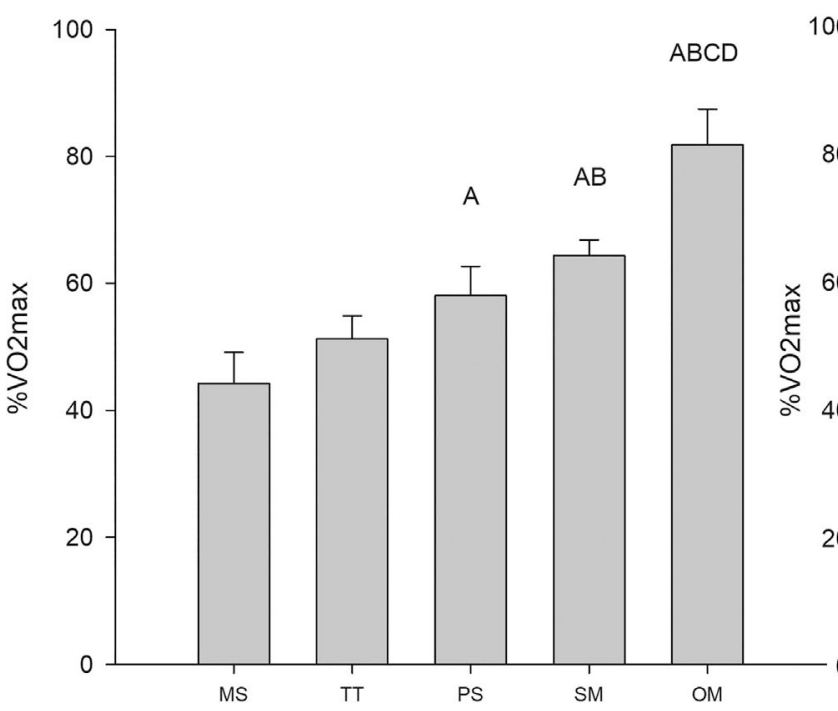

MALE

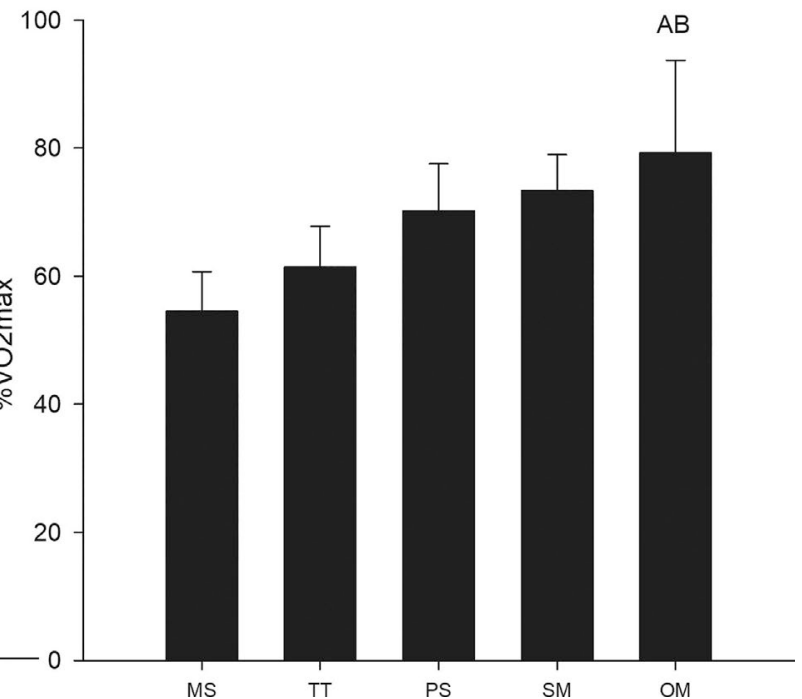

Figure 2 - \% $\mathrm{VO}_{2}$ max reached in the multi-shuttlecock, technical-tactical, physical, simulated match, and official match for the female and male athletes. A, significant difference compared to multi-shuttlecock; B, significant difference compared to technical-tactical; C, significant difference compared to physical; D, significant difference compared to simulated match $(\mathrm{p}<0.05)$. 


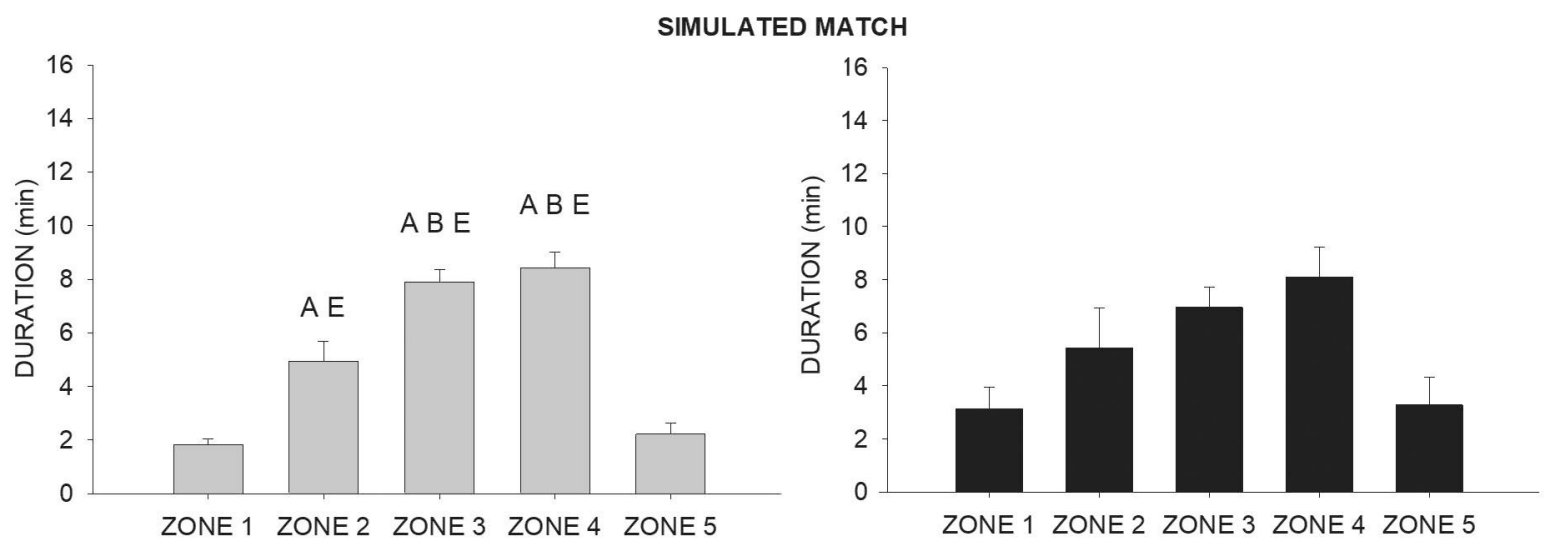

OFFICIAL MATCH
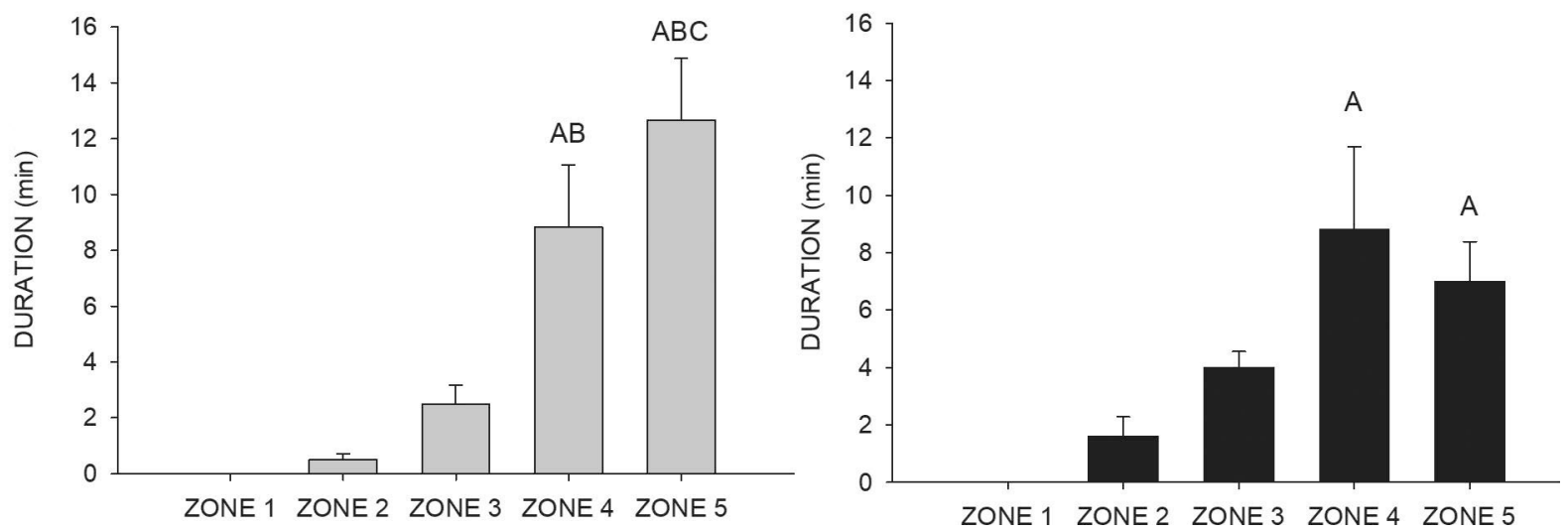

Figure 3 - Duration of time in the HR zones (min) reached in the multi-shuttlecock, technical-tactical, physical, simulated match, and official match for the female and male athletes. A, significant difference in the multi-shuttlecock training; B, significant difference in the technical-tactical training; C, significant difference compared to physical training; D, significant difference compared to the official matches $(\mathrm{p}<0.05)$.

performance ${ }^{24}$, did not alter the HR response and did not affect the physical performance of the participants.

\section{Discussion}

It is worth mentioning that no significant differences were found between the HRmax obtained in the $\mathrm{VO}_{2} \max$ test, training methods, and OM for male and female athletes. However, previous studies have observed higher HRmax during maximal exercise tests when compared to official badminton and tennis training and matches ${ }^{8,25}$. This may have occurred due to the differences between the controlled situation of the laboratory compared to training and $\mathrm{OM}$, such as specific movements, temperature, and external factors.

The $\mathrm{VO}_{2}$ max evaluation test used in this study can indirectly estimate the physiological demands of the training methods due to the linear positive correlation between $\mathrm{VO}_{2}$ and HR. However, this comparison can present relevant limitations due to the singularities of simulated and official matches when compared to a lab-based test ${ }^{26}$. Further studies should use different methods to evaluate the aerobic demand, such as indirect calorimetry.
For the female athletes, the intensities based on \% HRmax of OM $(86.79 \pm 4.13)$ found in our investigation were close to that of the OM assessed in junior $(89.2 \pm$ $4.1 \%)^{27}$ and adult female badminton athletes $(88.4 \pm$ $5.1 \%)^{28}$. This confirms the similar intensity found in the literature for both adult and junior athletes. Regarding the male players, the training methods resulted in lower \% HRmax than those of SM $(89 \pm 4.6 \%)^{28}$ and training 82.6 $\pm 4.5 \%{ }^{25}$ in adults. However, this comparison should be considered with caution, since no studies were found evaluating this variable with junior badminton athletes.

From our data, it was possible to identify that the training sessions and $\mathrm{OM}$ were performed at medium to high intensity (70-100\% HRmax). These results are similar to those of adult soccer athletes ${ }^{10}$, which confirms the high physical demand of badminton. Thus, a training protocol with adequate intensity is necessary in order to increase performance, as it was observed that badminton requires elevated aerobic fitness, $\mathrm{VO}_{2}$ max, and intermittent high-intensity performance in training sessions at $78-85 \% \mathrm{HRmax}^{20}$.

The $\mathrm{SM} \% \mathrm{VO}_{2} \max$ in the female players is similar to that of SM in adult women $(72.6 \pm 7.2 \%)^{28}$. The male 

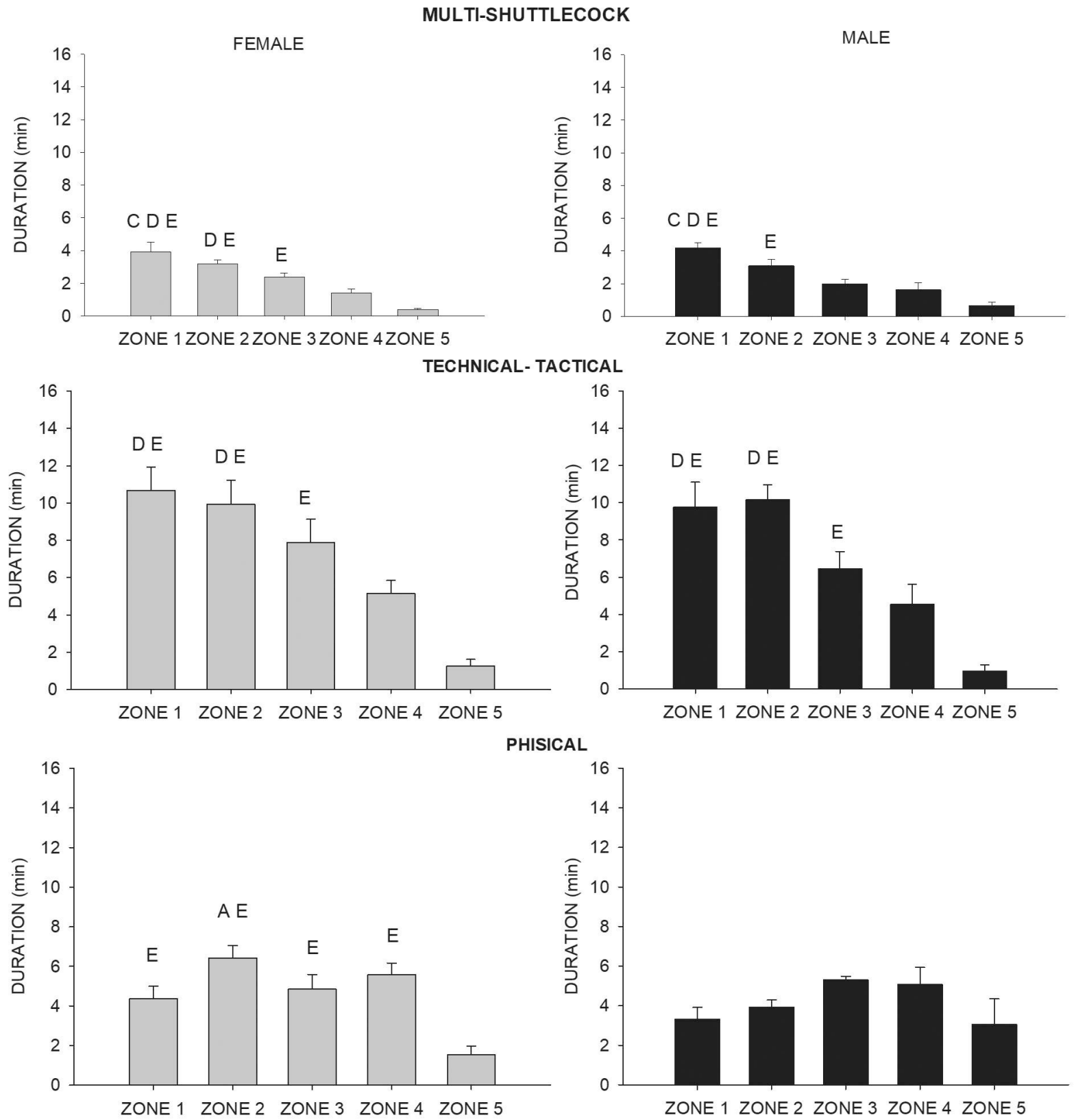

Figure 3 (cont.) - Duration of time in the HR zones (min) reached in the multi-shuttlecock, technical-tactical, physical, simulated match, and official match for the female and male athletes. A, significant difference in the multi-shuttlecock training; B, significant difference in the technical-tactical training; C, significant difference compared to physical training; D, significant difference compared to the official matches ( $\mathrm{p}<0.05$ ).

players of our study had similar $\% \mathrm{VO}_{2}$ max to those of a similar study ${ }^{28}$ for the SM and higher $\% \mathrm{VO}_{2} \max$ compared with the MS, TT, and PS values $(54.5 \pm 2.5 \%, 60.4$ $\pm 3.8 \%$, and $74.8 \pm 5.3 \%$, respectively) of another investigation $^{25}$. Considering that the aerobic system provides approximately $65 \%$ of the energy necessary for the maintenance of high- and moderate-intensity rallies, in addition to the short-time recovery ${ }^{28}$, the training intensity mentioned in our study can support athletes and coaches during training programming in order to achieve optimal performance in OMs.
Previous studies determined the HR intensity zones for elite badminton players as low $=<60 \%$ HRmax, average $=60.1-80 \%$ HRmax, and High $=>80.1 \%$ HRmax $^{29,30}$. Thus, in our study, we observed that MS is a low-intensity training method, TT has low to average intensity, and PS varies from low to high intensity. In contrast, male athletes achieved equivalent performance in all five zones during the MS, TT, and PS, which suggests that these training sessions range from low to high intensity. SM remained in medium to high intensities for the female athletes, while it was equally distributed through all zones 
Table 1 - Energy expenditure in kcal.min ${ }^{-1}$ in the MS, TT, PS, SM, and OM situations for female and male athletes.

\begin{tabular}{|c|c|c|}
\hline Situation & Female (kcal.min ${ }^{-1}$ ) & Male (kcal.min ${ }^{-1}$ ) \\
\hline MS & $5.96 \pm 0.99$ & $7.98 \pm 2.07$ \\
\hline TT & $6.89 \pm 0.87$ & $9.04 \pm 2.65$ \\
\hline PS & $7.79 \pm 0.76$ & $10.50 \pm 2.49$ \\
\hline SM & $8.64 \pm 0.63^{\mathrm{A}}$ & $11.10 \pm 3.18$ \\
\hline $\mathrm{OM}$ & $10.74 \pm 0.53^{\mathrm{ABCD}}$ & $14.87 \pm 4.63^{\mathrm{ABC}}$ \\
\hline
\end{tabular}

MS, multi-shuttlecock training; TT, tactical training; PS, physical training; SM, simulated match; OM, official match. A, significant difference compared to multi-shuttlecock; $\mathrm{B}$, significant difference compared to technical-tactical; $\mathrm{C}$, significant difference compared to physical; D, significant difference compared to simulated match $(\mathrm{p}<0.05)$.

for the male athletes. OM was verified to be a high-intensity exercise for both male and female athletes.

These differences may be due to neuromuscular, morphological, and metabolic differences between males and females. A meta-analysis study verified that male individuals show substantially better results in tests of muscle strength and cardiovascular fitness, although no significant differences in movement quality tests were found between males and females ${ }^{31}$. This demonstrates that differences in performance between men and women are to be expected, requiring adaptation of planning and training for each sex.

Our results are in agreement with those found in studies with soccer players ${ }^{10,14}$, which demonstrates that the players maintained a greater intensity during competition. This is due to external factors, such as motivation in OM and training-induced adaptations. Moreover, training methods can be managed during the training programming in order to reach a favorable performance at the competitive level. Moreover, the use of wearable technology allows more systematic monitoring of physiological and physical demands during training, and OM plays ${ }^{13}$. In fact, HR evaluation is regarded as a valid procedure to estimate the relative exercise intensity and guide the training interventions aimed at improving performance. Therefore, the individualized exercise prescription through \% HRmax is of great importance to guarantee precision and obtain results beneficial to performance and health ${ }^{32}$.

Our results differ from those of previous studies that assessed EE in recreational racquetball players $(11.1 \mathrm{kcal} /$ $\left.\min ^{-1}\right)^{33}$ in advanced and recreational tennis players $(263.1 \pm 49.4 \text { and } 281.3 \pm 61.8 \mathrm{kcal} / \mathrm{min} \text {, respectively })^{34}$, despite the similarities between them due to their intermittent nature. Such differences can be justified by the specificity in the movement actions and durations of each sport discipline. In addition, to date, no studies are verifying the intensity of badminton expressed in $\mathrm{kcal} / \mathrm{min}$ in junior athletes to allow for new comparisons.

In addition, the EE of the training sessions (MS, SM, TT, and PS) were lower than that of OM for both male and female players. During the specific training period, the athlete is expected to increase his level of technical-tactical, physical, and psychological proficiency, which enables maximum performance in the forthcoming competitions $^{35}$. Our data can be used by coaches and athletes to better guide their training protocols in order to achieve better results.

The use of wearable sensor technology is useful to measure the physiological demand in training and official badminton matches. Since training and real-match demands are different, different strategies of programming and adequacy of training load should be considered in order to enhance physical performance. Our data are helpful for exercise professionals to determine the intensity conducive to aerobic training adaptation.

Considering that the training sessions analyzed present different duration compared to the OM, our data regarding the specific intensity of each training method can be of great value for coaches and athletes. As the MS is the training method of the smallest intensity, it can be used in moments in which the technical training is prioritized. Similarly, as the SM is the most strenuous training method, it can be used in technical, tactical, and physical training periods.

This study will assist coaches and athletes in achieving optimal gains in training and better performance in OM plays. Thus, the parameters of all training methods should be used to create variation in training loads, whether they are tactical, technical, or physical. Further studies should investigate these variables under on-court conditions. This is because differences have been found between $\mathrm{HR}$ and $\mathrm{VO}_{2}$ when comparing on-court simulated matches and lab-based tests ${ }^{36}$. Therefore, a potential limitation of the current study is that all tests were carried out under controlled laboratory conditions and the training/ competition was carried out on the court.

\section{Conclusion}

The MS training presented less physiological demand and the OM had greater intensity. SM, on the other hand, had the closest physiological demand to that of OM. Thus, the parameters of all training methods must be used to create variation in training loads in tactical, technical, or physical training. Further studies should investigate cardiorespiratory fitness in field conditions as a potential limitation of the present study is that all $\mathrm{VO}_{2} \max$ tests were performed in laboratory-controlled conditions whereas training and official matches were performed on the court.

\section{Conflict of interest}

The authors declare no potential conflict of interest. 


\section{Acknowledgments}

We would like to thank all volunteers who participated in this study, as well as all staff from the Brazilian Confederation of Badminton, Federal University of Maranhão (UFMA), and the Federal University of Piauí (UFPI) for the technical assistance. This work was supported by the Maranhão Foundation for the Protection of Research and Scientific and Technological Development (FAPEMA) under grant no. BM-02684/17.

\section{References}

1 Deka P, Berg K, Harder J, Batelaan H, McGrath M. Oxygen cost and physiological responses of recreational badminton match play. J Sports Med Phys Fitness. 2017;57(6):760-5. doi:10.23736/S0022-4707.16.06319-2

2 Fitzsimons M, Dawson B, Ward D, Wilkinson A. Cycling and running tests of repeated sprint ability. Aust J Sci Med Sport. 1993;25(4):82-7.

3 Phomsoupha M, Laffaye G. The science of badminton: game characteristics, anthropometry, physiology, visual fitness, and biomechanics. Sport Med. 2015;45(4):473-95. doi:10.1007/s40279-014-0287-2

4 Chen H-L, Chang-Jun, Chen WUTC. Physiological and notational comparison of new and old scoring systems of singles matches in men's badminton. Asian J Phys Educ Recreat. 2011;17(1):6-17.

5 Laffaye G, Phomsoupha M, Dor F. Changes in the game characteristics of a badminton match: A longitudinal study through the Olympic game finals analysis in men's singles. J Sport Sci Med. 2015;14(3):584-90.

6 Zekan-Petrinović L. Badminton-unknown sport. Acta Med Croat. 2007;61(1):49-52.

7 Babolola J. Effects of 8-weeks Circuit Training Programme on Physiological and Performance Characteristics of University Racket Game Players. J Asian Sci Res. 2011;1 (4):143-9.

8 Ramos Álvarez JJ, Del Castillo Campos MJ, Polo Portes C, Ramón Rey M, Bosch Martín A. Analysis of the physiological parameters of junior spanish badminton players. Rev Int Med y Ciencias la Act Fis y del Deport. 2016;16(61):45-54. doi:10.15366/rimcafd2016.61.004

9 Angga PD. Anthropometric and motor performance of junior badminton athletes. Adv Heal Sci Res. 2019;7(Icssh 2018):143-6. doi:10.2991/icssh-18.2019.33

10 Coelho D, Rodrigues VM, Condessa LA, Mortimer LACF, Soares DD, Garcia ES. Intensidade de sessões de treinamento e jogos oficiais de futebol. Rev bras Educ Fís Esp. 2008;22(3):211-8. doi:10.1590/S1807-55092008000300005

11 Fernandez J, Fernandez-Garcia B, Mendez-Villanueva A, Garcia B, Terrados N. Exercise intensity in tennis: simulated match play versus training drills. Med Sci Tennis. 2005;10 (1):6-7.

12 Kilit B, ̈̈enel Ö, Arslan E, Can S. Physiological responses and match characteristics in professional tennis players during a one-hour simulated tennis match. J Hum Kinet. 2016;50(2):83-92. doi:10.1515/hukin-2015-0173
13 Montgomery PG, Pyne DB, Minahan CL. The physical and physiological demands of basketball training and competition. Int J Sports Physiol Perform. 2010;5(1):75-86. doi:10.1123/ijspp.5.1.75

14 Teixeira ML, Cabido CET, Coelho DB, Mendes TT. Intensidade de jogos e treinamentos de futebol na categoria sub- 15 Intensity. Rev Bras Futeb. 2014;6(2):60-8.

15 Moreno JH. Análisis de los parámetros espacio y tiempo en el fútbol sala. La distancia recorrida, el ritmo y dirección del desplazamiento del jugador durante un encuentro de competición: los casos de J.Gay (defensa), C. Marrero (cierre), J. Beto (pivote), J. Limon. Apunt Educ Física y Deport. 2001;62:32-44.

16 Sell KM, Ledesma AB. Heart rate and energy expenditure in division in field hockey players during competitive play. $\mathrm{J}$ Strength Cond Res. 2016;30(8):2122-8. doi:10.1519/JSC.0000000000001334

17 Silami-Garcia E, Espirito-Santo LC, Garcia AMC, Nunes VNG. Energy expenditure of professional soccer players during official games. Med Sci Sport Exerc. 2005;37(5):s87.

18 Lohman TG, Going S. AAssessmentof body composition and energy balance. In: Lamb D, Murray R, eds. Perspectives in Exercise Science and Sports Medicine. Cooper Publishing Group; 1998:61-105.

19 Castagna C, Belardinelli R, Impellizzeri FM, Abt GA, Coutts AJ, D'Ottavio S. Cardiovascular responses during recreational 5-a-side indoor-soccer. J Sci Med Sport. 2007;10(2):89-95. doi:10.1016/j.jsams.2006.05.010

20 Astrand P, Rodahl K, Dahl HA, Stromme SB. Tratado de Fisiologia do Trabalho: Bases Fisiológicas do Exercício. Artmed; 2006.

21 American College of Sports Medicine (ACSM). Guidelines for Exercise Testing and Prescription. 9th ed. Lippincott, Williams, and Wilkins; 2014.

22 Sawka M, Cheuvront S. Avaliação da hidratação dos atletas. Sport Sci Exerc. 2005;18(2).

23 Cohen J. Statistical Power Analysis for the Behavioral Sciences. 2nd ed. LEA; 1988.

24 Cardoso A, Moreira A, Paula C, Oliveira L, Baganha R, Dias R. Modulação nos níveis de hidratação após a prática do atletismo e performance de corrida. Rev Bras Nutr Esportiva. 2013;7(38):6.

25 Faccini P, Dai-Monte A. Demands of badminton match play. Am J Sport Med. 1996;24(6):64-6.

26 Achten J, Jeukendrup AE. Heart rate monitoring: Applications and limitations. Sport Med. 2003;33(7):517-38. doi:10.2165/00007256-200333070-00004

27 Fernandez-Fernandez J, Tellez JGA, Moya-Ramon M, Cabello-Manrique D, Mendez-Villanueva A. Gender differences in game responses during badminton match play. J Strength Cond Res. 2013;27(9):2396-404.

28 Faude O, Meyer T, Rosenberger F, Fries M, Huber G, Kindermann W. Physiological characteristics of badminton match play. Eur J Appl Physiol. 2007;100(4):479-85. doi:10.1007/s00421-007-0441-8

29 Alcock A, Cable N. A comparison of singles and doubles badminton: heart rate response, player profiles and game characteristics. Int J Perf Anal Spor. 2009;9(1):228-37.

30 Esposito F, Impellizzeri FM, Margonato V, Vanni R, Pizzini $\mathrm{G}$, Veicsteinas A. Validity of heart rate as an indicator of 
aerobic demand during soccer activities in amateur soccer players. Eur J Appl Physiol. 2004;93(1):167-72.

31 Courtright SH, McCormick BW, Postlethwaite BE, Reeves CJ, Mount MK. Meta-analysis of sex differences in physical ability: Revised estimates and strategies for reducing differences in selection contexts. J Appl Psychol. 2013;98(4):62341. doi: $10.1037 / \mathrm{a} 0033144$

32 Garber CE, Blissmer B, Deschenes MR, Franklin BA, Lamonte MJ, Lee IM, Nieman DC, Swain DP; American College of Sports Medicine. Quantity and quality of exercise for developing and maintaining cardiorespiratory, musculoskeletal, and neuromotor fitness in apparently healthy adults: Guidance for prescribing exercise. Med Sci Sports Exerc. 2011;43(7):1334-59. doi:10.1249/MSS.0b013e318213fefb

33 Berg K, Narazaki K, Latin R, Vincent W, Meisinger M, Sjoberg C, Kaufman C. Oxygen cost and energy expenditure of racquetball. J Sports Med Phys Fitness. 2007;47(4):395-400.

34 Fernandez-Fernandez J, Sanz-Rivas D, Sanchez-Muñoz C, Pluim B, Tiemessen I, Mendez-Villanueva A. A comparison of the activity profile and physiological demands between advanced and recreational veteran tennis players. J Strength Cond Res. 2006;20(3):477-82.

35 Dantas EHM. A Prática Da Preparação Física. 3rd ed. Shape; 1995.
36 Rampichini S, Limonta E, Pugliese L, Cè E, Bisconti AV, Gianfelici A, La Torre A, Esposito F. Heart rate and pulmonary oxygen uptake response in professional badminton players: comparison between on-court game simulation and laboratory exercise testing. Eur J Appl Physiol. 2018;118 (11):2339-47. doi:10.1007/s00421-018-3960-6

\section{Corresponding author}

Karen Christie Gomes Sales, Universidade Federal do Maranhão, Departamento de Educação Física, São Luís, MA, Brasil.

E-mail: karenchristie1@hotmail.com.

Manuscript received on November 11, 2020

Manuscript accepted on April 8, 2021

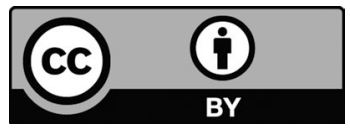

Motriz. The Journal of Physical Education. UNESP. Rio Claro, SP, Brazil - eISSN: 1980-6574 - under a license Creative Commons - Version 4.0 